\title{
Über fluorierte Phosphate, Sulfate, Selenate, Tellurate und Dithionate.
}

\section{Von}

R. F. Weinluand und J. Alfa.

In Gemeinschaft mit 0 . LaUEnsteis ${ }^{1}$ hatte der eine von uns beobachtet, dafs in den Alkalijodaten 1 Atom Sauerstoff durch 2 Atome Fluor ersetzbar ist.

Im Anschlufs hieran haben wir nun untersucht, $o b$ auch in den Salzen der Metalloidsäuren der 5. und 6. Gruppe der Elemente Sauerstoff durch Fluor vertretbar sei. Dabei fanden wir, dals auch bei einigen von diesen $z$ war nicht Sauerstoffatame, aber doch Hydroxylgruppen durch Fluor substituiert werden können, speziell konnte eine solche teilweise Vertretbarkeit von Hydroxylgruppen durch Fluor bei den Phosphaten, Sulfaten, Selenaten, Telluraten und Dithionaten einiger Alkalimetalle nachgewiesen werden.

Betreffs der Darstellung dieser fluorierten Salze sei bemerkt, dafs sie durchgehend durch Einwirkung von Flulssäure auf die betreffenden Sauerstoffsalze erhalten werden.

\section{A. Fluorphosphate. ${ }^{2}$}

1. Monokaliummonofluorphosphat, $\mathrm{P}(\mathrm{OH})_{3}(\mathrm{OK}) \mathrm{Fl}$.

Zur Darstellung dieses Salzes löst man den Verdampfungsrückstand einer wässerigen Lösung von $1 \mathrm{Mol}$. Trikaliumphosphat und $1 \mathrm{Mol}$. Kaliumhydroxyd in soviel Fluorwasserstoffsäure von etwa $40 \%$, dafs die heifse Flüssigkeit - die Lösung erfolgt unter starker Erhitzung

1 R. F. Weinland und O. Lauenstein, Z. anorg. Chem. 20, 30.

2 Die Beobachtung der Fluorphosphate und Fluorsulfate wurde in den Ber. deutsch. chem. Ges. 1898, 123, als vorläufige Mitteilung veröffentlicht. 
- beim Erkalten nicht sogleich Salz ausscheidet. Man konzentriert dann bei ganz mälsiger Wärme, worauf das Salz in der Kälte auskrystallisiert. Die Ausbeute ist klein: etwa $40 \mathrm{~g}$ Phosphat liefern nur 4-5 g Salz. Hat man zu weit eingedampft und löst man dann das sich ausscheidende Salzgemenge durch erneuten Zusatz von Fluorwasserstoffsäure, so erhält man das Salz beim Konzentrieren nicht mehr oder nur in sehr geringer Menge.

Auch auf folgende Weise lärst sich das Salz darstellen: Man trägt in eine mittels eines Kältegemisches stark abgekühlte Lösung von 2 Mol. Kaliumhydroxyd in überschüssiger $40 \%$ iger Fluorwasserstoffsäure ungeachtet der Ausscheidung von sauerem Kaliumfluorid 1 Mol. Phosphorpentoxyd in kleinen Anteilen ein. Die hierauf bei mäfsiger Wärme konz. Flüssigkeit liefert beim Stehen in einem mit Paraffin ausgekleideten und mit Ätzkalk beschickten Exsiccator das Salz in verhältnismälsig guter Ausbeute. Besonders schnell beginnt die Krystallisation nach dem Impfen der Lösung mit einem Krystall des Salzes.

Durch Behandeln von tertiärem, sekundärem oder primärem Kaliumphosphat mit Fluorwasserstoffsäure erhält man jedoch das Salz nicht.

Es lälst sich aus Fluorwasserstoffsäure von etwa $40 \%$ umkrystăllisieren, wenn man die Lösung in einem, wie oben angegeben, mit Ätzkalk beschickten Exsiccator und nicht an der Luft verdunsten lälst; aber man erhält nur sehr wenig des gelösten Salzes in guter Ausbildung zurïck.

Das Salz bildet farblose, glänzende, dicke, annähernd rechtwinkelig viereckige Tafeln von bis zu. $5 \mathrm{~mm}$ Kantenlänge. Herr Prof. Dr. P. Grotir war so gütig, eine krystallographische Untersuchtng des Salzes durch Herrn Dr. H. ZirnarebL ausführen zu lassen. Die Untersuchung war im vorliegenden Falle dadurch sehr erschwert, dals sich die Krystalle an der Luft rasch trübten; trot\%dem gelang es Herrn Dr. Zirngiebi ${ }_{L}$, ein sicheres Ergebnis zu erhalten.

,Krystallsystem: monoklin holoedr.

$$
\begin{aligned}
a: b: c & =0.8501: 1: 0.6268 \\
\beta & =78^{\circ} 55^{\prime} .
\end{aligned}
$$

Beobachtete Formen: $a(100) ; c(001) ; s(\overline{101}) ; m(110) ; b(010) ; t(021)$; $p(121)$.

Die Reflexe sind alle mittelmälsig, besonders diejenigen von $t(021)$ und $p$ (121), deren Indices sich aus dem Zonenverband ergaben. 


$$
\begin{aligned}
\text { Beobachtet: } & \text { Berechnet } \\
a c=(100):(001)={ }^{*} 78^{\circ} 55^{\prime} & - \\
c s=(001):(\overline{101})={ }^{*} 40^{\circ} 8^{\prime} & - \\
m m=(110):(\overline{110})={ }^{*} 100^{\circ} 20^{\prime} & - \\
c m=(001):(110)=81^{\circ} 27^{\prime} & 81^{\circ} 31^{\prime}
\end{aligned}
$$

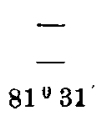

Spaltbarkeit nicht beobachtet.

Axenebene $=$ Symmetrieebene.

Ätzfiguren lassen keine Hemimorphie erkennen."

In trockener Luft ist das Salz ziemlich beständig, doch greift es auch hierin Glas allmählich an. In feuchter Luft werden die Krystalle unter Verlust von Fluorwasserstoff rasch trübe. In Paraffin behalten sie ihre Durchsichtigkeit einige Zeit. Beim Erhitzen schmilzt das Salz, es verflüchtigen sich Wasser und Fluorwasserstoff, und Kaliummetaphosphat bleibt zurück. In Wasser löst sich das Salz zu einer stark fluorwasserstoffsauren Flüssigkeit; engt man diese ein, so wird es nicht wieder erhalten. Beim Erwärmen des Salzes mit Schwefelsäure entwickelt sich Fluorwasserstoff. (Es sei noch erwähnt, dafs bei dem Salze die Reaktion auf Phosphorsäure mit Ammoniummolybdänat durch die Gegenwart des Fluorwasserstoffes stark beeinträchtigt wird.)

\section{Analyse.}

Das Kaliumfluorphosphat und die entsprechende Rubidium- und Cäsiumverbindung wurden auf folgende Weise analysieut:

Die Phosphorsäure wurde nach Vertreibung des Fluorwasserstoffes durch Erwärmen des Salzes mit konz. Schwefelsäure mit Magnesiamischung gefällt. - Zur Bestimmung des Alkalis wurde nach Verflüchtigung des Fluorwasserstoffes durch Erwärmen mit Schwefelsäure die Phosphorsäure mittels Eisenchlorids entfernt, das Filtrat eingedampft und das Alkali als Sulfat gewogen.

Das Fluor wurde nach der etwas modifizierten Restmethode von WöнLER, durch Verflüchtigung als Siliciumfluorid, bestimmt. Der hierzu konstruierte Apparat war dem Morr'schen Kohlensäurebestimmungsapparat' ähnlich. Er bestand aus einem etwa $70 \mathrm{cem}$ fassenden Glaskolben, welcher mit drei Öffnungen versehen war; die eine, seitliche, zum Einbringen der Substanz dienende, war durch einen eingeschliffenen Glasstopfen verschlossen; in die andere war ein etwa $20 \mathrm{ccm}$ fassender Scheidetrichter zur Aufnahme der Schwefelsäure cingeschliffen; an die dritte Öffuung war ein Glasröhrchen angeschmolzen, welches das sich entwickelnde Siliciumfluorid zunächst in eine etwa $4 \mathrm{ccm}$ grofse Glaskngel und aus dieser in ein birnförmiges Glasgefäls führte, welches letztere

${ }^{1}$ Eine Abbildung davon findet șich in Fruserros, Quantit. Analysc (6. Aufl.) 1, 447. 
mit Bimssteinstïckchen gefüllt war; diese waren mit konz. Kupfersulfatlösung getränkt und bei $240^{\circ}$ getrocknet worden. Die beiden letzteren Gefăfse dienen zum Zurückhalten sich verflüchtigender Schwefelsäuredämpfe. Nachdem das Salz, gemengt mit der mindestens zehnfachen Menge feinen, ausgeglühten Quarzpulvers, ${ }^{2}$ in den .Kolben gebracht, und der Scheidetrichter zu $4 / 5$ mit konz. Schwefelsäure gefüllt war, wurde der Apparat gewogen und dann die Schwefelsäure langsam zu dem Gemenge einfliefsen gelassen. Durch Bewegung des Kolbens wurde die Substanz in der Schwefelsäure verteilt. Der Kolben wurde hierauf auf einer grofsen Asbestplatte solange auf $150^{\circ}$ erwärmt und gleichzeitig ein langsamer Strom sorgfältig getrockneter Luft durch den Apparat gedrückt, bis die austretende Luft keine Dämpfe mehr enthielt, welche feuchtes Lackmuspapier röteten. Um die Temperatur zu messen, war neben dem Apparat ein Kölbchen mit konz. Sehwefelsäure und einem Thermometer aufgestellt. Aufserdem wurde die leere Glaskugel und das Gefäls mit dem Bimssteinstückchen durch mehrere übereinandergelegte Asbestplatten vor der Erwärmung durch die hei §se, aufsteigende Luft geschützt. Nachdem der Apparat erkaltet war, wurde zurückgewogen: Die Differenz war die Menge des gebildeten Siliciumfluorids. Hiervon wurden dann noch $1.5 \mathrm{mg}$ abgezogen, ein Verlust, welchen der Apparat, wie durch eine Reihe blinder Versuche festgestellt wurde, durch Verdampfen der Schwefelsäure bei 3-4 stündigem Erhitzen auf $150^{\circ}$ erleidet.

Die erhaltenen Resultate waren für den vorliegenden Zweck hinreichend genau. Fehler entstehen einerseits durch den Wassergehalt dex Salze, andererseits dadurch, dals mit dem auf Filtrierpapier von der Mutterlauge befreiten Fluorsalz etwas organische Substanz in das Gefäls gelangt, welche reduzierend auf die Schwefelsäure einwirkt.

Der Wassergehalt der Fluorphosphate wurde durch Glïhen derselben mit Bleioxyd und Auffangen des verflüchtigten Wassers in einem Calciumchloridrohr bestimmt.

I. $0.3307 \mathrm{~g}$ Substanz lieferten $0.2370 \mathrm{~g} \mathrm{P}_{2} \mathrm{O}_{7} \mathrm{Mg}_{2}=19.95 \% \mathrm{P}$.

\begin{tabular}{|c|c|c|c|c|}
\hline 1.0119, & $n$ & $"$ & $0.5769,, \mathrm{SO}_{4} \mathrm{~K}_{2}$ & $=25.6 \% \mathrm{~K}$ \\
\hline 0.5104, & $"$ & " & $\begin{array}{l}\text { nach } \mathrm{Abzug} \text { von } \\
0.0859 \mathrm{~g} \mathrm{SiFl}_{4}\end{array}$ & $\begin{array}{l}1.5 \mathrm{mg} \\
=12.2 \% \mathrm{FL} .\end{array}$ \\
\hline 0.4229, & $"$ & $"$ & $0.0726, \mathrm{H}_{2} \mathrm{O}$ & $=17.2 \% \quad \mathrm{H}_{2} \mathrm{O}$. \\
\hline 0.3199, & $"$ & $"$ & $0.2295, \mathrm{P}_{2} \mathrm{O}_{7} \mathrm{Mg}_{2}$ & $=20.00 \% \mathrm{P}$ \\
\hline 0.7988, & $"$ & $"$ & $0.4427, \mathrm{SO}_{4} \mathrm{~K}_{2}$ & $=24.9 \% \mathrm{~K}$. \\
\hline 0.6360 & $"$ & $"$ & $\begin{array}{l}\text { nach Abzug von } \\
0.1021 \mathrm{~g} \mathrm{SiFl}_{4}\end{array}$ & $\begin{array}{l}1.5 \mathrm{mg} \\
=11.7 \% \mathrm{Fl} .\end{array}$ \\
\hline 0.3472, & $"$ & " & $0.0620, \mathrm{H}_{2} \mathrm{O}$ & $=17.8 \% \quad \mathrm{H}_{2} \mathrm{O}$. \\
\hline
\end{tabular}

III. $0.6478 \mathrm{~g}$ eines aus Kaliumfluorid und Phosphorpentoxyd dargestellten Salzes lieferten $0.4650 \mathrm{~g} \mathrm{P}_{2} \mathrm{O}_{7} \mathrm{Mg}_{2}=20.00 \% \mathrm{P}$.

$0.5998 \mathrm{~g}$ desselben Salzes lieferten nach Abzug von $1.5 \mathrm{mg}$ $0.0973 \mathrm{~g} \mathrm{SiFl}_{4}=11.8 \% \mathrm{Fl}$.

1 Wirft man zum starken Glühen erhitzte Quarzkörner in kaltes Wasser, so lassen sie sich leicht zu Pulver zerreiben. 


\begin{tabular}{|c|c|c|c|c|}
\hline \multirow{2}{*}{\multicolumn{2}{|c|}{$\begin{array}{l}\text { Berechnet für } \\
\mathrm{P}(\mathrm{OH})_{s}(\mathrm{OK}) \mathrm{Fl} \text { : }\end{array}$}} & \multicolumn{3}{|c|}{ Gefunden: } \\
\hline & & I. & II. & III. \\
\hline $\mathrm{P}$ & 19.85 & 19.95 & 20.00 & 20.00 \\
\hline $\mathbf{K}$ & 25.06 & 25.6 & 24.9 & $\ldots$ \\
\hline Fl & 12.15 & 12.2 & 11.7 & 11.8 \\
\hline $\mathrm{H}_{2} \mathrm{O}$ & 17.33 & 17.2 & 17.8 & - \\
\hline $\mathrm{O}$ & 25.61 & 25.05 & 25.6 (a. d Diff.) & - \\
\hline & $100.00 \%$ & $100.0 \%$ & $100.0 \%$ & \\
\hline
\end{tabular}

\section{Monorubidiummonofluorphosphat, $\mathrm{P}(\mathrm{OH})_{3}(\mathrm{ORb}) \mathrm{Fl}$.}

Das Rubidiumfluorphosphat ist dem Kaliumsalz völlig analog. Man erhält es, wie dieses, durch Auflösen des Verdampfungsrückstandes einer wässerigen Lösung von $2 \mathrm{Mol}$. Trirubidiumphosphat und 1 Mol. Rubidiumkarbonat in $40 \%$ iger Fluorwasserstoffsäure und vorsichtige Konzentration der Lösung. Die Ausbeute ist jedoch gröfser als beim Kaliumsalz. Es läfst sich auch aus Rubidiumfluorid und Phosphorpentoxyd nach der beim Kaliumfluorphosphat angegebenen Methode darstellen.

Das Rubidiumfluorphosphat zeigt dieselbe Krystallform wie das Kaliumfluorphosphat. Herr Dr. ZIRNGIEBL teilte uns hierüber folgendes freundlichst mit:

„Die Krystalle gleichen denen des Kaliumsalzes und zeigen auch ähnliche Winkel, geben aber so schlechte und abweichende Messungen, dals eine Feststellung des Axenverhältnisses unmöglich ist. Der Prismenwinkel schwankt zwischen 101-105\%. Die beobachteten Formen sind: $a(100) ; \quad c(001) ; s(\overline{101}) ; m(110) ; \quad b(010)$; $u(210)$ ?:

Auch im übrigen verhält es sich durchaus wie das Kaliumsalz.

$$
\text { Analyse. }
$$

I. $0.5440 \mathrm{~g}$ Substanz lieferten $0.2987 \mathrm{~g} \mathrm{P}_{2} \mathrm{O}_{7} \mathrm{Mg}_{2}=15.3 \% \mathrm{P}$.

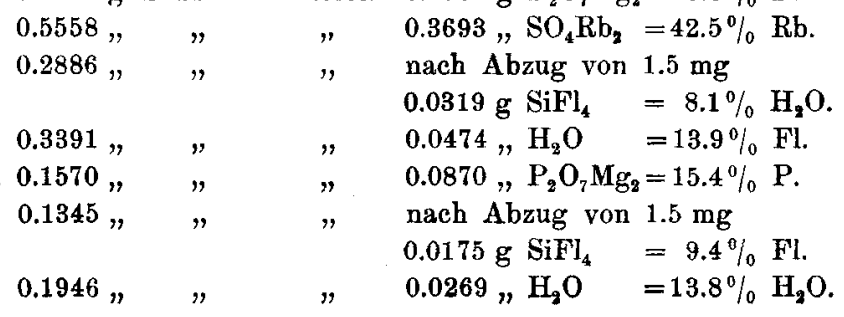




\begin{tabular}{lccc}
\multicolumn{2}{c}{ Berechnet für } & \multicolumn{2}{c}{ Gefunden: } \\
\multicolumn{2}{c}{$\mathrm{P}(\mathrm{OH})_{3}(\mathrm{ORb}) \mathrm{Fl}:$} & l. & II. \\
$\mathrm{P}$ & 15.33 & 15.3 & 15.4 \\
$\mathrm{Rb}$ & 42.20 & 42.5 & - \\
$\mathrm{Fl}$ & 9.37 & 8.1 & 9.4 \\
$\mathrm{H}_{2} \mathrm{O}$ & 13.35 & 13.9 & 13.8 \\
$\mathrm{O}$ & 19.75 & $20.2($ a. d. Diff. $)$ & - \\
\multicolumn{2}{c}{$100.00 \%$} & $100.00 \%$ &
\end{tabular}

3. Monocäsiummonofluorphosphat, $\mathrm{P}(\mathrm{OH})_{3}(\mathrm{OCs}) \mathrm{Fl}$.

Verdampft man eine Lösung von 2 Mol. Tricäsiumphosphat und $1 \mathrm{Mol}$. Cäsiumkarbonat zur Trockene und nimmt den Rückstand in überschüssiger Fluorwasserstoffsäure auf, so scheidet sich nach dem Einengen der Lösung das den beschriebenen Salzen entsprechende Cäsiumfluorphosphat aus. Es ist indessen bei diesem viel schwieriger als bei jenem, gute Krystalle zu erhalten, und man mufs den richtigen Konzentrationsgrad genau herausfinden. Lälst man die noch zu verdünnte Lösung an der Luft verdunsten, so krystallisieren fluorfreie Phosphate aus. Man vermeidet dies durch Verdunstenlassen der Lösung in einem mit Ätzkalk gefüllten Exsiccator.

Das Cäsiumfluorphosphat entspricht nach Form und Eigenschaften vollständig der analogen Kalium- und Rubidiumverbindung.

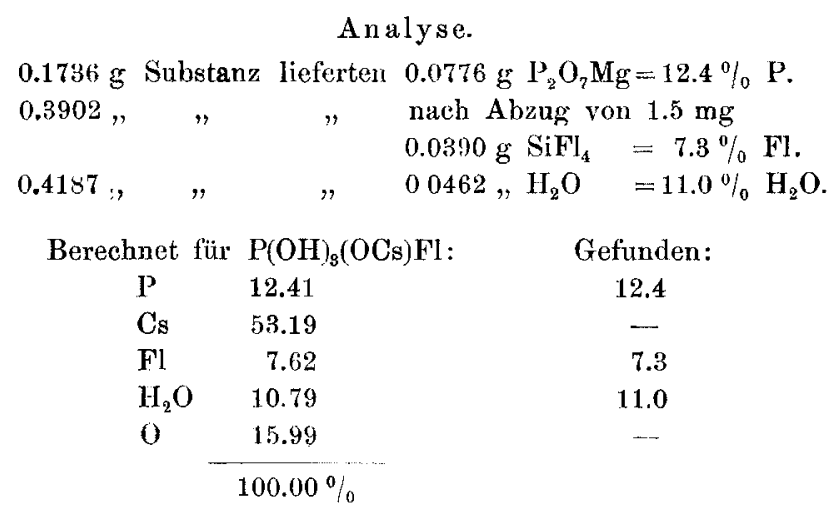

Eine entsprechende Natrium- oder Ammoniumverbindung nach den beschriebenen Methoden darzustellen, gelang nicht. Auch aus einer Lösung von Silberphosphat in Fluorwasserstoffsäure scheidet sich dieses fluorfrei (krystallinisch) wieder aus. Desgleichen liefsen sich Phosphate $\mathrm{z}$ wei- und dreiwertiger 
Metalle auf diese Weise nicht fluorieren (nachgewiesen beim Calcium-, Nickel-, Kobalt-, Mangan-, Aluminium- und Ferriphosphat).

Was die Konstitution der Fluorphosphate betrifft, so lassen sie sich wohl am einfachsten von primärem Phosphat durch Anlagerung von $1 \mathrm{Mol}$. Fluorwasserstoff unter Aufrichtung des doppelt an den Phosphor gebundenen Sauerstoffatoms ableiten:<smiles>O=[PH](O)(O)O</smiles>

Monokaliumphosphat.

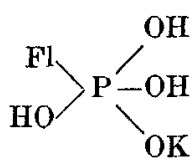

Monokaliummonofluorphosphat.

In dem vollständig hydroxyliert gedachten primären Phosphat - $\mathrm{P}(\mathrm{OH})_{4}(\mathrm{OK})$ - wäre demnach eine Hydroxylgruppe durch Fluor ersetzt; demgemäls wurden die Salze als Monofluorphosphate bezeichnet.

Die Frage, ob das Wasser der Salze verschieden fest gebunden sei, und daher bei ihrem Erhitzen sich bei verschiedenen Temperaturen verflüchtige, was an sich wahrscheinlich ist, konnte durch den Versuch nicht eindeutig beantwortet werden. Da nämlich die Salze, für sich allein erhitzt, aufser Wasser auch "Fluorwasserstoff verlieren, müssen sie, mit Zinkoxyd oder Bleioxyd gemischt, erhitzt werden. Diese entreissen aber einmal dem Salze sein Fluor, und dann verbinden sie sich mit dem primären Phosphat, so dals das sich verflüchtigende Wasser nicht nur das im Molekül als neutrales Hydroxyl funktionierende Wasser, sondern auch Neutralisationswasser vorstellt. Die Menge des letzteren ist aber unter anderem von der mehr oder weniger innigen Mischung des Salzes mit dem Oxyd abhängig. Thatsächlich lieferten auch die Versuche mit dem Kaliumfluorphosphat, bei denen dieses mit Zinkoxyd auf $110^{\circ}$ erhitzt wurde, schwankende Wassermengen. Dazu kommt noch, dals das entstehende Zinkfluorid, da es für gewöhnlich mit 4 Mol. Wasser krystallisiert, wahrscheinlich aus dem Salze Wasser an sich reilst; wie aber durch besondere Versuche festgestellt wurde, giebt das Zinkfluorid, $\mathrm{ZnFl}_{2} \cdot 4 \mathrm{H}_{2} \mathrm{O}$, sein Wasser nur sehr schwer ab. - Beim Erhitzen des Kaliumfluorphosphats mit Bleioxyd zeigte sich bei etwa $150^{\circ}$, als noch lange nicht alles Wasser sich verflüchtigt hatte, bei allen Versuchen eine Gewichtszunahme, deren Ursache nicht aufgefunden werden konnte.

Es wurde schliefslich noch versucht, durch Einwirkung höchst konzentrierter (etwa $70 \%$ iger) Fluorwasserstoffsäure auf das 
Kaliummonofluorphosphat ein noch fluorreicheres Salz darzustellen. Aber der nach dem Konzentrieren der Lösung zurückbleibende Syrup lieferte auch bei der Behandlung mit alkoholischer Fluorwasserstoffsäure keinen einheitlichen Körper.

Es sei noch erwähnt, dafs BERTHELOT ${ }^{1}$ schon früher aus der Existenz einer fluorphosphorigen Säure auf die einer Fluorphosphorsäure geschlossen hat. Die Bildung der ersteren folgerte er aus der bei der Zersetzung von Phosphortrifluorid mit Alkalien auftretenden Wärmetönung. - Auch Moissan ${ }^{2}$ fand, dafs Phosphortrifluorid von Alkalien nur unvollständig zersetzt wird infolge Bildung einer fluorphosphorigen Säure.

\section{B. Fluorsulfate.}

\section{Trikaliumdifluordisulfat, $\mathrm{S}_{2} \mathrm{O}_{7} \mathrm{Fl}_{2} \mathrm{~K}_{3} \mathrm{H} \cdot \mathrm{H}_{2} \mathrm{O}$.}

Dieses Salz scheidet sich aus einer Lösung von neutralem oder primärem Kaliumsulfat in $40 \%$ iger Fluorwasserstoffsäure beim Konzentrieren bei mälsiger Wärme in schönen Krystallen und in sehr guter Ausbeute aus. Auch aus einer mit Kaliumfluorid versetzten Lösung von neutralem Kaliumsulfat in Fluorwasserstoffsäure erhält man dieses Salz. Die Krystalle werden mit Fluorwasserstoffsäure abgewaschen und nach dem Pressen zwischen Filtrierpapier kurze Zeit über Ätzkalk getrocknet; eventuell können sie aus Fluorwasserstoffsäure umkrystallisiert werden.

Das Salz bildet farblose, durchsichtige, bis $2 \mathrm{~cm}$ lange, flache Prismen. Die Krystalle treten teils einzeln auf (selten), teils vereinigen sie sich $\mathrm{zu}$ schwalbenschwanzartigen $\mathrm{Z}$ willingen, teils bilden sie, flach auf einander liegend, geschichtete Aggregate von ziemlich grofser Ausdehnung. Herr Dr. ZinngiebL war so freundlich, auch diese Krystalle zu messen; er berichtet darüber folgendes:

$$
\begin{gathered}
\text { „Krystallsystem: monoklin sphen. } \\
\qquad: b: c=1.013: 1: 0.8218 \\
\beta=71^{\circ} 21^{\prime} .
\end{gathered}
$$

Beobachtete Formen: $a(100) ; m(110) ; n(130) ; c(001) ; s(\overline{101})$ und einige nicht bestimmbare Sphenoïde und Domen.

Die Reflexc sind mittelmäfsig von den Flächen der Zonen (010) und (001). Dagegen geben einige Sphenoïde und Domen, welche noch vorhanden sind, gar keine Reflexe.

1 Berthelot, Ann. Chim. Phys. [6] 6, 358; Jahresber. von Fittica 1855, 443.

${ }^{2}$ Moissan, Ann. Chim. Phys. [6] 6, 468; Jahresber. von Firtuca 1885, 440. 


$\begin{array}{rlr}\text { Beobachtet: } & \text { Berechnet: } \\ a c=(100):(001)={ }^{*} 71^{\circ} 21^{\prime} & - \\ a m=(100):(110)=45^{\circ} 53^{\prime} & 46^{\circ} 12^{\prime} \\ a n=(100):(130)={ }^{*} 72^{\circ} 17^{\prime} & - \\ a s=(100):(\overline{101})={ }^{*} 62^{\circ} 35^{\prime} & - \\ c n=(001):(130)=76^{\circ} 51^{\prime} & 77^{\circ} 8^{\prime}\end{array}$

Axenebene $\perp$ Symmetrieebene. Auf $a(100)$ ist ein Axenbild (spitze Bisectrix) gegen $e(001)$ wenig geneigt sichtbar. Dispersion $\varrho<v$, stark. Doppelbrechung ziemlich sehwach. Axenwinkel ca. $70^{\circ}$ in Luft für Na-Licht."

In trockenem Zustand ist das Salz ziemlich beständig und verliert auch bei kurzer Aufbewahrung im Exsiccator kein Wasser. Doch greift es Glas bei längerer Berührung damit an. An der Luft verlieren die Krystalle schnell ihren Glasglanz infolge der durch den Wasserdampf derselben bewirkten Bildung von Sulfat und Fluorwasserstoff.

Beim Erhitzen schmilzt das Salz, und es verflüchtigen sich zuerst Wasser und Fluorwasserstoff; bei stärkerem Erhitzen entweicht Schwefelsäure und im Rückstand bleibt Kaliumsulfat.

Das Kaliumfluorsulfat ist in Wasser unter Zersetzung - Abspaltung des Fluors als Fluorwasserstoff - leicht löslich. 'Konzentriert man die stark sauere Lösung, so erhält man das Salz nicht mehr; dagegen lälst es sich, wie erwähnt, aus Fluorwasserstoffsäure der angegehenen Konzentration sehr gut umkrystallisieren.

Beim Erwärmen des Salzes mit konz. Schwefelsäure entwickelt sich reichlich Fluorwasserstoff.

Analyse.

Das Kaliumfluorsulfat und das analoge Rubidium- und Cäsiumsalz wurden auf folgende Weise analysiert:

Die Schwefelsäure wurde nach Vertreibung des Fluorwasserstoffes durch wiederholtes Abrauchen des Salzes mit konz. Salzsäure mit Baryumchlorid gefällt.

Zur Bestimmung des Alkalis warden die Salze mit wenig Schwefelsäure versetzt, diese und der Fluorwasserstoff durch Erwärmen vertrieben, und der Rückstand geglüht.

Das Fluor wurde wie bei den Phosphaten (S. 45) bestimmt. Aufserdem konnte der Gehalt an Fluor, nachdem das Verhältnis zwischen Schwefelsăure und Alkali festgestellt worden war, durch Titration der bei der Zersetzung des Salzes durch Wasser frei werdenden Säuremenge ermittelt werden. $\mathrm{SO}_{4}$ und Alkalimetall verhalten sich in den Salzen wie 2:3, folglich wird bei der Titration der freien Säure aufser der gebildeten Fluorwasserstoffsäure noch der vierte Teil der vorhandenen Schwefelsäure gefunden. Die diesem entsprechende Anzahl Kubikcentimeter Alkali ist von der verbrauchten Anzahl abzuziehen, 
der Rest entspricht dem Fluorwasserstoff. Es wurde mit $1 / 10^{-n o r m . ~ A l k a l i ~ t i t r i e r t . ~}$ Die Resultate waren sehr genau.

Die Berechnung des Fluorgehaltes bei dieser Bestimmungsmethode gestaltete sich wesentlich einfacher, nachdem durch andere Fluorbestimmungen die Zusammensetzung der Salze als der Formel $\mathrm{S}_{2} \mathrm{O}_{7} \mathrm{Fl}_{2} \mathrm{Me}_{8} \mathrm{H}_{3} \mathrm{H}_{2} \mathrm{O}$ entsprechend gefunden worden war. Unter dem Einflufs von Wasser zersetzen sich nun diese Salze nach der Gleichung:

$$
\mathrm{S}_{2} \mathrm{O}_{7} \mathrm{Fl}_{2} \mathrm{Me}_{3} \mathrm{H}+\mathrm{H}_{2} \mathrm{O}=\mathrm{SO}_{4} \mathrm{Me}_{2}+\mathrm{SO}_{4} \mathrm{MeH}+2 \mathrm{HFl} \text {. }
$$

Von der zur Neutralisation der freiwerdenden Säure nötigen Anzahl Kubikcentimeter Alkali ist somit der dritte Teil, als der Schwefelsäure entsprechend, abzuziehen, der Rest kommt dem Fluorwasserstoff zu.

Das Wasser wurde wie bei den Fluorphosphaten durch Glühen der Salze mit Bleioxyd bestimmt.

I. $0.5813 \mathrm{~g}$ Substanz lieferten $0.7772 \mathrm{~g} \mathrm{BaSO}=18.4 \% \mathrm{~S}$.

$$
\begin{aligned}
& 0.3914, \quad " \quad " \quad 0.2944, \mathrm{~K}_{2} \mathrm{SO}_{4}=33.7 \% \mathrm{~K} \text {. } \\
& 0.3729, \quad \text { " } " \quad \text { nach Abzug von } 1.5 \mathrm{mg} \\
& 0.0495 \mathrm{~g} \mathrm{SiFl}_{4}=9.7 \% \text { Fl. } \\
& 0.2760 " \quad " \quad 0.0227, \mathrm{H}_{2} \mathrm{O}=8.2 \% \mathrm{H}_{2} \mathrm{O} \text {. }
\end{aligned}
$$

\begin{tabular}{|c|c|c|c|}
\hline $0.3202 "$ & $"$ & $"$ & $0.2367, \mathrm{~K}_{2} \mathrm{SO}_{4}=33.2 \% \mathrm{~K}$ \\
\hline 0.4861 & " & $"$ & $\begin{array}{l}\text { nach Abzug von } 1.5 \mathrm{mg} \\
0.0741, \mathrm{SiFl}_{4}=11.1 \% \text { Fl. }\end{array}$ \\
\hline .104 & , & " & $0.0119, \mathrm{H}_{2} \mathrm{O}$ \\
\hline
\end{tabular}

II. $0.3374 \mathrm{~g}$ Substanz lieferten $0.4513 \mathrm{~g} \mathrm{BaSO}_{4}=18.4 \% \mathrm{~S}$.

III. $0.2603 \mathrm{~g}$ Substanz verbrauchten zur Bindung der freien Säure der wässerigen Lösung $22.4 \mathrm{ccm} \mathrm{1/10-norm.} \mathrm{Kalilauge.} \mathrm{Der} \mathrm{vorhandenen}$ Schwefelsäure entsprechen $7.5 \mathrm{ccm} 1 / 10$-norm. Kalilauge, der Rest, $14.9 \mathrm{ccm}$, kommt dem Fluorwasserstoff zu $=10.87 \% \mathrm{Fl}$.

IV. $0.3674 \mathrm{~g}$ Substanz verbrauchten unter denselben Umständen $31.2 \mathrm{ccm}$ $1 / 10^{-n o r m}$. Kalilauge; $1 / \mathrm{s}$ hiervon, $10.4 \mathrm{ccm}$, entspricht der Schwefelsäure, der Rest, $20.8 \mathrm{ccm}$, kommt dem Fluorwasserstoff zu

$$
=10.85 \% \text { Fl. }
$$

V. $0.2059 \mathrm{~g}$ Substanz verbrauchten ebenso $17.3 \mathrm{ccm} 1 / 10^{-n o r m}$. Kalilauge; $1 / \mathrm{s}$ hiervon, $5.7 \mathrm{ccm}$, entspricht der Schwefelsäure, der Rest, $11.6 \mathrm{ccm}$,

\begin{tabular}{|c|c|c|c|c|c|c|c|}
\hline \multicolumn{2}{|c|}{$\mathrm{S}_{2} \mathrm{O}_{7} \mathrm{Fl}_{2} \mathrm{~K}_{8} \mathrm{H} . \mathrm{H}_{2} \mathrm{O}:$} & I. & II. & III. & IV. & V. & \multirow[t]{2}{*}{ VI. } \\
\hline $\mathbf{S}$ & 18.29 & 18.4 & 18.4 & & & & \\
\hline K & 33.49 & 33.7 & 33.2 & & & & \\
\hline Fl & 10.84 & 9.7 & 11.1 & 10.87 & 10.85 & 10.70 & 10.85 \\
\hline $\mathrm{H}_{2} \mathrm{O}$ & 7.71 & 8.2 & 7.8 & & & & \\
\hline 0 & 29.67 & \multicolumn{4}{|c|}{30.0 (a.d.D.) 29.5 (a. d. Diff.) } & & \\
\hline & $100.00 \%$ & $1.10 .0 \%$ & 100.0 & & & & \\
\hline
\end{tabular}
kommt dem Fluorwasserstoff $\mathrm{zu} \quad=10.70 \%$ Fl.

VI. $0.3105 \mathrm{~g}$ Substanz verbrauchten ebenso $26.5 \mathrm{ecm} 1 / 10^{-n o r m}$. Kalilauge; $1 / \mathrm{s}$ hiervon, $8.8 \mathrm{ccm}$, entspricht der Schwefelsäure, der Rest, $17.7 \mathrm{ccm}$, kommt dem Fluorwasserstoff zu $\quad=10.85 \%$ Fl.

Bereehnet für 
2. Trirubidiumdifluordisulfat, $\mathrm{S}_{2} \mathrm{O}_{7} \mathrm{Fl}_{2} \mathrm{Rb}_{3} \mathrm{H} \cdot \mathrm{H}_{2} \mathrm{O}$.

Das Rubidiumfluorsulfat bildet sich wie das Kaliumsalz und stimmt auch in Form und Eigenschaften völlig mit jenem überein.

\section{Analyse.}

\begin{tabular}{|c|c|c|c|}
\hline $0.3334 \mathrm{~g}$ & Substanz & lieferten & $0.3219 \mathrm{~g} \mathrm{BaSO}_{4}=13.2 \% \mathrm{~S}$. \\
\hline 0.4246, & $"$ & $"$ & $0.3476, \mathrm{Rb}_{3} \mathrm{SO}_{4}=52.4 \% \mathrm{Rb}$ \\
\hline 0.1574, & $"$ & " & $0.009, \mathrm{H}_{2} \mathrm{O}=5.7 \% \quad \mathrm{H}_{2} \mathrm{O}$. \\
\hline 0.3568, & $n$ & $"$ & $\begin{array}{l}\text { nach Abzug von } 1.5 \mathrm{mg} \\
0.0345 \mathrm{~g} \mathrm{SiFl}_{\mathrm{t}}=7.0 \% \mathrm{Fl}\end{array}$ \\
\hline
\end{tabular}

\begin{tabular}{ccc} 
Berechnet für $\mathrm{S}_{2} \mathrm{O}_{7} \mathrm{Fl}_{2} \mathrm{Rb}_{3} \mathrm{H} . \mathrm{H}_{2} \mathrm{O}:$ & Gefunden: \\
$\mathrm{S}$ & $\mathbf{1 3 . 1 2}$ & 13.2 \\
$\mathrm{Rb}$ & 52.36 & 52.4 \\
Fl & 7.76 & 7.0 \\
$\mathrm{H}_{2} \mathrm{O}$ & 5.52 & 5.7 \\
$\mathrm{O}$ & 21.24 & 21.7 (a. d. Diff.) \\
\cline { 2 - 3 } & $100.00 \%$ & $100.0 \%$
\end{tabular}

\section{Tricäsiumdifluordisulfat, $\mathrm{S}_{8} \mathrm{O}_{7} \mathrm{Fl}_{2} \mathrm{Cs}_{8} \mathrm{H}_{2} \mathrm{H}_{2} \mathrm{O}$.}

Das Cäsiumfluorsulfat lälst sich wie die beschriebenen Salze darstellen, aber es ist, wie bei dem Cäsiumfluorphosphat (S. 48) schwieriger, ein gut krystallisiertes Salz zu erhalten und man mufs hierzu, wie dort angegeben, verfahren. Im übrigen entspricht es völlig dem Rubidium- und Kaliumsalz.

\section{Analyse.}

$$
\begin{aligned}
& 0.2524 \mathrm{~g} \text { Substanz lieferten } 0.2152 \mathrm{~g} \mathrm{Cs}_{2} \mathrm{SO}_{4}=62.8 \% \mathrm{Cs} . \\
& 0.3103 " \Rightarrow \quad " \quad 0.2289, \mathrm{BaSO}_{4}=10.1 \% \mathrm{~S} \text {. } " \text { verbrauchten zur Neutralisation der freien Säure }
\end{aligned}
$$

der wässerigen Lösung $10.5 \mathrm{ccm} 1 / 10$-norm. Kalilauge; $1 / \mathrm{s}$ hiervon, $3.5 \mathrm{ccm}$, entspricht der Sehwefelsäure, der Rest, $7.0 \mathrm{ccm}$, kommt dem Fluorwasserstoff zu $\begin{aligned} & 0.3372 \mathrm{~g} \text { Substanz lieferten } 0.0149 \mathrm{~g} \mathrm{H}_{2} \mathrm{O}=6.0 \% \mathrm{Fl} . \\ &=4.4 \% \mathrm{H}_{2} \mathrm{O} .\end{aligned}$

\begin{tabular}{ccc} 
Berechet für $\mathrm{S}_{2} \mathrm{O}_{7} \mathrm{Fl}_{2} \mathrm{Cs}_{8} \mathrm{H} . \mathrm{H}_{2} \mathrm{O}:$ & Gefunden: \\
$\mathrm{S}$ & 10.14 & 10.1 \\
$\mathrm{Cs}$ & 63.11 & 62.8 \\
$\mathrm{Fl}$ & 6.03 & 6.0 \\
$\mathrm{H}_{2} \mathrm{O}$ & 4.27 & 4.4 \\
$\mathrm{O}$ & 16.45 & 16.7 (a. d. Diff.) \\
\cline { 2 - 3 } & $100.00 \%$ & $100.0 \%$
\end{tabular}


Fluorierte Sulfate vom Natrium oder Ammonium oder einem mehrwertigen Metall liefsen sich auf ảie angegebene Weise nicht darstellen, was, wie erwähnt, auch bei den Phosphaten nicht möglich war.

Die beschriebenen Fluorsulfate sind Derivate des saueren Alkalisulfats der Formel $\mathrm{SO}_{4} \mathrm{Me}_{2}, \mathrm{SO}_{4} \mathrm{MeH}$ oder $\left(\mathrm{SO}_{4}\right)_{2} \mathrm{Me}_{3} \mathrm{H}$, von denen das Kaliumsalz sich bekanntlich beim Umkrystallisieren von Monokaliumsulfat aus Wasser bildet. Dieses sauere Sulfat geht durch Aufnahme von $2 \mathrm{Mol}$. Fluorwasserstoff in das Fluorsulfat über:

$$
\left(\mathrm{SO}_{4}\right)_{2} \mathrm{Me}_{3} \mathrm{H}+2 \mathrm{HFl}=\mathrm{S}_{2} \mathrm{O}_{7} \mathrm{Fl}_{2} \mathrm{Me}_{3} \mathrm{H} . \mathrm{H}_{2} \mathrm{O} .
$$

Formuliert man das sauere Alkalisulfat $\left(\mathrm{SO}_{4}\right)_{2} \mathrm{Me}_{3} \mathrm{H}$ folgendermalsen:

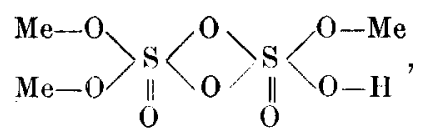

und dies ist wohl seine natürlichste Konstitutionsformel, so befindet sich an jedem Schwefelatom noch je ein doppelt an dieses gebundenes Sauerstoffatom; werden beide durch Hinzutritt von je 1 Mol. Fluorwasserstoff hydroxyliert, so erhält man die Fluorsulfate: ${ }^{1}$

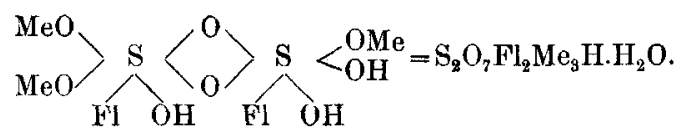

Diese Auffassung der Fluorsulfate ist wohl die einfachste Annahme über ihre Konstitution. Sieht man hiervon ab, so kann man die Salze auch als Doppelsalze von 1 Mol. sauerem Alkalifluorid mit $2 \mathrm{Mol}$. primärem Alkalisulfat formulieren:

\section{$\mathrm{MeFl}_{2} \mathrm{H} .2 \mathrm{SO}_{4} \mathrm{MeH}$.}

Die Versuche, die über das höchst wahrscheinlich verschiedene Verhalten des Wassers beim Erhitzen dieser Salze angestellt wurden, führten aus den bei den Phosphaten (S. 49) angeführten Gründen auch hier zu keinem sicheren Ergebnis. Beim Erhitzen des Kaliumfluorsulfats mit Zinkoxyd verflüchtigten sich bei $110^{\circ}$ annähernd $2 / 3$ des vorhandenen Wassers (gefunden: $4.3 \% ; 4.4 \%$; $4.7 \%$; berechnet: $5.14 \%$, also etwa der in der Formel als

${ }^{1}$ Im Text wurde des leichteren Überblickes halber die Formel $\mathrm{S}_{8} \mathrm{O}_{7} \mathrm{Fl}_{2} \mathrm{Me}_{8} \mathrm{H} 1 . \mathrm{H}_{2} \mathrm{O}$ benutzt, obwohl sie das Wasser als Krystallwasser er. scheinen lärst. 
neutrales Hydroxyl funktionierende Teil, während der Rest, der in der Formel als saueres Hydroxyl auftritt, erst bei $190^{\circ}$ entweicht.

\section{Fluorselenate.}

Von Fluorselenaten konnte ein den Fluorsulfaten analoges Kalium- und Rubidiumsalz und aufserdem ein Ammoniumsalz von etwas anderer Zusammensetzung beobachtet werden; ein Cäsiumsalz liefs sich nicht darstellen. Man erhält indessen die Fluorselenate im Gegensatz zu den Fluorsulfaten, welche sich unter mannigfachen Umständen bilden, nur unter Einhaltung ganz bestimmter Bedingungen.

\section{Trikaliumdifluordiselenat, $\mathrm{Se}_{2} \mathrm{O}_{7} \mathrm{Fl}_{2} \mathrm{~K}_{3} \mathrm{H} \cdot \mathrm{H}_{2} \mathrm{O}$.}

Bei den ersten Versuchen wurde eine bei mälsiger Temperatur konz. Lösung von Kaliumselenat ${ }^{1}$ in überschülsiger $40 \%$ iger Fluorwasserstoffsäure behufs Krystallisation an der Luft stehen gelassen; dabei schied sich nach längerer Zeit das Kaliumselenat fluorfrei aus. Als jedoch die eingeengte Lösung in einen Exsiccator über Ätzkalk gebracht wurde, schieden sich im Laufe mehrerer Wochen winzige Kryställchen aus, welche den qualitativen Reaktionen zufolge ein fluorhaltiges Selenat vorstellten. Mit diesen wurde nun eine neue Lösung geimpft und auf diese Weise eine reichliche und schöne Krystallauscheidung erhalten. Die Analyse des Salzes zeigte, dafs ein dem Kaliumtluorsulfat entsprechendes Selenat vorlag. Besonders wichtig ist die Konzentration der zu impfenden Lösung; bringt man sie zu verdünnt in den Exsiccator, so kriecht der gröfste Teil davon unter Krustenbildung über den

1 Die zu diesen Versuchen benutzte Sclensäure wurde auf folgende, verhältnismäfsig rasch zu einer reinen Selensäurelösung führeuden Weise dargestellt: Eine Lösung von Selen in Salpetersäure wird mit Natriumkarbonat stark alkalisch gemacht, und nach eventucller Filtration - bei der Neutralisation der salpetersaueren Lösung findet meist eine flockige Ausscheidung vermutlich von Metallkarbonaten oder hydroxyden statt - zur Trockene verdampft. Der Rückstand wird mit Salpeter und Soda so lange vorsichtig geschmolzen, als sich Stickstoffoxyde entwickeln. Die Schmelze wird in Wasser gelöst, und die kalte, mit Salzsäure schwach angesäuerte Lösung mit Baryumchlorid gefüllt. Das gewaschene und getrocknete Baryumselenat wird hierauf mit etwas weniger als der berechneten Menge stark verdünnter Schwefelsäure einige Zeit gekocht, wodurch man eine völlig schwefelsäurefreie Lösung von Selensäure erhält. Aus dieser wurden durch Neutralisation mit den betreffenden Karbonaten die Selenate dargestellt. 
Rand der Platinschale, und es bleibt nur eine breiige Masse darin zurück. Hat man jedoch den richtigen Konzentrationsgrad getroffen, so beginnt kurze Zeit nach der Impfung die Krystallisation.

Die Krystalle des Kaliumfluorselenats gleichen denen des Kaliumfluorsulfats (S. 50). Sie sind indessen bedeutend kleiner als jene nnd treten auch nur einzeln auf. Obgleich die Krystalle an der Luft rasch verwittern, gelang es Herrn Dr. ZrangIebL doch, folgende Winkel annähernd zu messen:

$$
\begin{aligned}
a c & =(100):(001)=71^{\circ} \quad(\mathrm{ca} .) \\
a m & =(100):(110)=45.5^{\circ}, " \\
a n & =(100):(130)=73^{\circ} \quad " \\
a s & =(100):(\overline{1} 01)=59^{\circ} \quad "
\end{aligned}
$$

Diese Werte liegen nahe bei denen des Kaliumfluorsulfats.

Wegen seiner geringen Neigung zur Krystallisation wurde vermutet, dafs das Salz hygroskopisch sein werde, es erwies sich aber, wie bereits erwähnt, als an der Luft ziemlich rasch verwitternd unter Abgabe von Wasser und Fluorwasserstoff. Infolgedessen greift es auch Glas rasch an. In Paraffin ist es einige Zeit haltbar. Erhitzt man das Salz im Reagensrohr, so schmilzt es, während sich Wasser und Fluorwasserstoff verflüchtigen; bei weiterem Erhitzen zersetzt sich die nicht völlig vom Alkali neutralisierte Selensäure in Selendioxyd, Sauerstoff und Wasser, welche entweichen, während Selenat im Rückstand bleibt.

In Wasser löst sich das Salz sehr leicht unter Zersetzung zu einer stark saueren Flüssigkeit.

\section{Analyse.}

Dic Fluorselenate wurden folgendermalsen analysiert:

Die Selensäure wurde nach Vertreibung des Fluorwasserstoffes durch mehrmaliges Abrauchen der Salze mit konz. Salpetersäure als Baryumselenat bestimmt. Durch Zusatz von Alkohol (etwa $5 \%$ zu der Fällungsflüssigkeit wurde die bestehende geringe Löslichkeit des Baryumselenats in Wasser sehr vermindert. Man stellt dann längere Zeit beiseite. Das in geringsem Überschufs zugesetzte Baryumehlorid wird durch den Alkohol in dieser Verdünnung nicht mitgefällt. Man wäscht den Niederschlag mit alkoholhaltigem Wasser. Das nach Entfernung des Baryums mit Schwefelsäure abgedampfte Filtrat lieferte das Alkalisulfat.

Nachdem das Verhältnis zwischen Selensäure und Alkali festgestellt war, konnte durch Titration der bei der Zersetzung der Salze durch Wasser gebildeten freien Säure mit $1 / 10$-norm. Alkali der Gehalt an Fluor ermittelt werden. Anch hier gab diese Methode sehr gute Resultate. (Wendet man 1/10-norm. Ammoniak an, so kann man in derselben Salzmenge noch die Selensäure und das Alkali bestimmen.) 
Der Wassergehalt wurde wie bei den Phosphaten (S. 46) und Sulfaten durch Glühen der Salze mit Bleioxyd ermittelt.

I. $0.3670 \mathrm{~g}$ Substanz lieferten $0.4645 \mathrm{~g} \mathrm{SeO}_{4} \mathrm{Ba}=35.7 \%$ Se.

Dieselbe Substanz lieferte $0.2139, \mathrm{SO}_{4} \mathrm{~K}_{2}=26.2 \% \mathrm{~K}$.

verbrauchte zur Bindung der freien Säure der wässerigen Lösung $24.8 \mathrm{~cm}{ }^{1} / 10^{-n o r m}$. Ammoniak; $1 / \mathrm{s}$ hiervon, $8.26 \mathrm{ccm}$, entspricht der Selensäure, der Rest, $16.54 \mathrm{ccm}$, kommt dem Fluorwasserstoff $\mathrm{zu} \quad=8.57 \% \mathrm{Fl}$. $0.3779 \mathrm{~g}$ Substanz lieferten $0.0235 \mathrm{~g} \mathrm{H}_{2} \mathrm{O}=6.2 \% \mathrm{H}_{2} \mathrm{O}$.

II. $0.8205, \quad " \quad, \quad 1.0275, \mathrm{SeO}_{4} \mathrm{Ba}=35.4 \% \mathrm{Se}$.

Dieselbe Substanz lieferte $0.4850, \mathrm{SO}_{4} \mathrm{~K}_{2}=26.5 \% \mathrm{~K}$.

$" \quad, \quad$ verbrauchte zur Bindung der freien Säure der wässerigen Lösung $54.9 \mathrm{~cm} 1 / 10^{-n o r m}$. Ammoniak; $1 / \mathrm{s}$ hiervon $718.3 \mathrm{ccm}$, entsprechen der Selensäure, der Rest, $36.6 \mathrm{ccm}$, kommt dem Fluorwasserstoff $\mathrm{zu} \quad=8.47 \% \mathrm{Fl}$.

Berechnet für

\begin{tabular}{lccc}
\multicolumn{2}{c}{$\mathrm{Se}_{2} \mathrm{O}_{7} \mathrm{Fl}_{2} \mathrm{~K}_{3} \mathrm{H} . \mathrm{H}_{2} \mathrm{O}:$} & I. & II. \\
$\mathrm{Se}$ & 35.58 & 35.7 & 35.4 \\
$\mathrm{~K}$ & 26.41 & 26.2 & 26.5 \\
$\mathrm{Fl}$ & 8.54 & 8.57 & 8.47 \\
$\mathrm{H}_{9} \mathrm{O}$ & 6.08 & 6.2 & - \\
$\mathrm{O}$ & 23.39 & 23.33 a. d. D. & - \\
\cline { 2 - 5 } & $100.00 \%$ & $100.0 \%$ &
\end{tabular}

2. Trirubidiumdifluordiselenat, $\mathrm{Se}_{2} \mathrm{O}_{7} \mathrm{Fl}_{2} \mathrm{Rb}_{3} \mathrm{H} \cdot \mathrm{H}_{2} \mathrm{O}$.

Das Rubidiumfluorselenat gleicht in jeder Beziehung dem beschriebenen Kaliumsalze, nur zeigt es eine etwas gröfsere Krystallisationsfähigkeit.

\section{Analyse.}

I. $0.3087 \mathrm{~g}$ Substanz lieferten $0.2978 \mathrm{~g} \mathrm{SeO}_{4} \mathrm{Ba}=27.2 \%$ Se. Dieselbe Substanz lieferte $0.2100, \mathrm{SO}_{4} \mathrm{Rb}_{2}=43.5 \% \mathrm{Rb}$.

" " verbrauchte zur Bindung der freien Säure der wässerigen Lösung $16.24 \mathrm{ccm} \mathrm{1/10-norm.} \mathrm{Ammoniak;} 1 / 3$ hiervon, $5.41 \mathrm{ccm}$, entspricht der Selensäure; der Rest, $10.83 \mathrm{ccm}$, der Fluorwasserstoffsäure $\quad=6.60 \% \mathrm{Fl}$. $0.6977 \mathrm{~g}$ Substanz lieferten $0.0332 \mathrm{~g} \mathrm{H}_{2} \mathrm{O}=4.8 \% \mathrm{H}_{2} \mathrm{O}$.

II. $0.3728, \quad, \quad, \quad 0.3642, \mathrm{SeO}_{4} \mathrm{Ba}=27.6 \% \mathrm{Se}$

Dieselbe Substanz verbrauchte bei der Titration der freien Säure der wässerigen Lösung $19.2 \mathrm{ccm} \mathrm{1/10-norm.} \mathrm{Ammoniak;} \mathrm{hiervon} \mathrm{ent-}$ sprechen $2 / 3,12.8 \mathrm{ccm}$, der Fluorwasserstoffsäure

$$
=6.52 \% \mathrm{Fl}
$$

III. $0.3927 \mathrm{~g}$ Substanz lieferten $0.3733 \mathrm{~g} \mathrm{SeO}_{4} \mathrm{Ba}=26.9 \% \mathrm{Se}$.

Dieselbe Substanz verbrauchte bei der Titration der freien Säure der wässerigen Lösung $20.2 \mathrm{ccm} 1 / 10$-norm. Ammoniak, wovon $2 / 3$, $13.47 \mathrm{ccm}$, der Fluorwasserstoffisäure entsprechen

$$
=6.51 \% \mathrm{Fl} \text {. }
$$




\begin{tabular}{|c|c|c|c|c|}
\hline & \multicolumn{3}{|l|}{-58} \\
\hline \multicolumn{2}{|c|}{ Berechnet für } & \multicolumn{3}{|c|}{ Gefunden: } \\
\hline \multicolumn{2}{|c|}{$\mathrm{Se}_{2} \mathrm{O}_{7} \mathrm{Fl}_{2} \mathrm{Rb}_{3} \mathrm{H} \cdot \mathrm{H}_{2} \mathrm{O}$} & I. & II. & III. \\
\hline Se & 27.10 & 27.2 & 27.6 & 26.9 \\
\hline $\mathrm{Rb}$ & 43.93 & 43.5 & -- & - \\
\hline $\mathrm{Fl}$ & 6.51 & 6.6 & 6.52 & 6.51 \\
\hline $\mathrm{H}_{2} \mathrm{O}$ & 4.63 & 4.8 & - & - \\
\hline 0 & 17.83 & \multicolumn{3}{|c|}{17.9 (a. d. Diff.) } \\
\hline & $100.00 \%$ & \multicolumn{3}{|c|}{$100.0 \%$} \\
\hline
\end{tabular}

Bei den Versuchen, ein fluoriertes Cäsiumselenat darzustellen, wurde stets nur ein schmieriger Krystallbrei erhalten, der beim Aufstreichen auf Filtrierpapier äufserst rasch zerflofs. Auch Impfen mit dem Rubidiumsalz veranlafste keine bessere Krystallisation. Vom Natrium liefs sich gleichfalls kein fluoriertes Selenat darstellen.

\section{Ammoniummonofluorselenat, $\mathrm{SeO}_{3}(\mathrm{OH}) \mathrm{Fl}\left(\mathrm{NH}_{4}\right)_{2}$.}

Man löst zur Darstellung dieses Salzes Ammoniumselenat in überschüssiger Fluorwasserstoffsäure und konzentriert die Lösung bei sehr mälsiger Wärme. Im Exsiccator über Ätzkalk scheidet sich dann das Ammoniumfluorselenat aus. Auch bei diesem Salz ist es nötig, durch Ausprobieren die richtige Konzentration herauszufinden, da die zu weit eingedampfte Lösung nur saueres Ammoniumfluorid liefert. Die erste, sehr geringe Ausscheidung des Salzes wurde nach einer Impfung der Lösung mit einem Rubidiumfluorselenatkrystall beobachtet. Mit Hilfe dieser Kryställchen war es möglich, in kurzer Zeit aus einer neuen Lösung das Ammoniumfluorselenat in schön ausgebildeten Krystallen zu erhalten.

Das Ammoniumfluorselenat bildet dem Kaliumfluorselenat ähnliche, flache Prismen. Über die Krystallform teilt uns Herr Dr. ZirngiebI folgendes freundlichst mit:

$$
\text { ,Krystallsystem: rhombisch (?). }
$$

Beobachtete Formen: $a(100) ; b(010) ; s(011)$.

Der Winkel, welchen $s(011)$ mit $b(010)$ bildet, konnte nur angenähert. im Mikroskop gemessen werden zu ca. $32.5^{\circ}$

Axenebene (010), auf $a$ tritt die stumpfe Bisectrix aus."

Das völlig trockene Salz verliert an der Luft seinen Glanz ziemlich rasch. Es ist nicht hygroskopisch, aber in Wasser sehr leicht unter Zersetzung zu einer stark fluorwasserstoffsaueren Flüssigkeit löslich. 


\section{Analyse.}

Das Fluor wurde bei diesem Salze wiederum durch Titration der bei seiner Zersetzung durch Wasser gebildeten freien Säure bestimmt. Da in diesem Falle Selensäure und Ammonium im Verhältnis des neutralen Salzes stehen, kommt die gesamte freie Säure auf Rechnung des Fluorwasserstoffes.

Der Wassergehalt wurde in der Art ermittelt, dals das Salz mit Bleioxyd im Rohr geglüht, und das ausgetriebene Wasser und Ammoniak in Schwefelsäure (in einem U-Rohr) aufgefangen wurden. Von der Gewichtszunahme wurde dann das (anderweitig bestimmte) Ammoniak abgezogen. Da sich mit dem Ammoniak und dem Wasser auch stets etwas Fluorwasserstoff verflüchtigte, fielen die Resultate etwas zu grofs aus.

I. $0.2880 \mathrm{~g}$ Substanz lieferten $0.3980 \mathrm{~g} \mathrm{SeO}_{4} \mathrm{Ba}=39.0 \%$ Se.

$0.2516, \quad " \quad, \quad$ beim Erhitzen mit Kalilauge eine $25 \mathrm{ccm}$

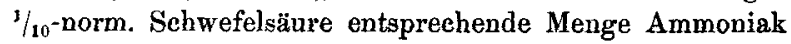

$$
=17.0 \% \mathrm{NH}_{2} \text {. }
$$

$0.4193 \mathrm{~g}$ Substanz verbrauchten zur Bindung der freien Säiure der wässerigen Lösung $20.9 \mathrm{ccm} 1 / 10$-norm. Ammoniak $=9.47 \% \mathrm{Fl}$.

$0.1444 \mathrm{~g}$ Substanz lieferten beim Erhitzen mit Bleioxyd $0.0455 \mathrm{~g}$ $\mathrm{NH}_{3}+\mathrm{H}_{2} \mathrm{O}$; die in der Substanz vorhandene Menge $\mathrm{NH}_{3}$ beträgt $0.0244 \mathrm{~g}$, der Rest, $0.0211 \mathrm{~g}$, ist Wasser $=14.6 \% \mathrm{H}_{2} \mathrm{O}$.

II. $0.2983 \mathrm{~g}$ Substanz lieferten $0.4189 \mathrm{~g} \mathrm{SeO} \mathrm{Ba}=39.6 \% \mathrm{Se}$.

Dieselbe Substanz verbrauchte zur Bindung der freien Säure der wä́sserigen Lösung $14.8 \mathrm{ccm} 1 / 10$-norm. Ammoniak $=9.42 \%$ Fl.

$0.2556 \mathrm{~g}$ Substanz lieferten beim Erhitzen mit Bleioxyd $0.0808 \mathrm{~g}$ $\mathrm{NH}_{3}+\mathrm{H}_{2} \mathrm{O}$; die in der Substanz enthaltene Menge $\mathrm{NH}_{3}$ beträgt $0.0437 \mathrm{~g}$, der Rest, $0.0371 \mathrm{~g}$, ist Wasser $=14.5 \% \mathrm{H}_{2} \mathrm{O}$.

III. $0.3111 \mathrm{~g}$ Substanz lieferten beim Erhitzen mit Bleioxyd $0,0971 \mathrm{~g}$ $\mathrm{NH}_{3}+\mathrm{H}_{2} \mathrm{O}$; das in der Substanz enthaltene Ammoniak beträgt $0.0532 \mathrm{~g}$, der Rest, $0.0439 \mathrm{~g}$, ist Wasser $=14.1 \% \mathrm{H}_{2} \mathrm{O}$.

\begin{tabular}{|c|c|c|c|}
\hline Berechnet für & \multicolumn{3}{|c|}{ Gefunden: } \\
\hline $\mathrm{O}_{3}(\mathrm{OH}) \mathrm{Fl}\left(\mathrm{NH}_{4}\right)_{2}$ & I. & II. & III. \\
\hline 39.69 & 39.0 & 39.6 & - \\
\hline 17.12 & 17.0 & - & 一 \\
\hline 9.54 & 9.47 & 9.42 & - \\
\hline 13.57 & 14.6 & 14.5 & 14.1 \\
\hline 20.08 & \multicolumn{3}{|c|}{19.93 (a. d. Diff.) } \\
\hline $100.00 \%$ & 100.0 & & \\
\hline
\end{tabular}

Die Konstitution des Kalium- und Rubidiumfluorselenats ${ }^{1}$ wird man als dem gleich zusammengesetzten Fluorsulfat (S. 54) entsprechend annehmen können:

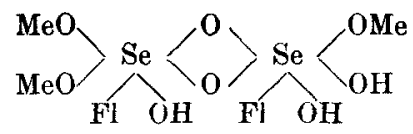

' Der Übersichtlichkeit halber wurde auch hier, wie bei den Fluorsulfaten, im Text die Formel $\mathrm{Se}_{2} \mathrm{O}_{7} \mathrm{Fl}_{4} \mathrm{Me}_{8} \mathrm{H} . \mathrm{H}_{2} \mathrm{O}$ benutzt. 
Das Ammoniumfluorselenat, $\mathrm{SeO}_{3}(\mathrm{OH}) \mathrm{Fl}\left(\mathrm{NH}_{4}\right)_{2}$, läfst sich am einfachsten als ein Ammoniumselenat ansehen, an das sich 1 Mol. Fluorwasserstoff unter Aufrichtung eines doppelt an das Selen gebundenen Sauerstoffatoms angelagert hat:<smiles>NO[Se](=O)(O[Na])O[Na]</smiles>

Ammoniumselenat,<smiles>[H][R5]([H])(O)O[Na]</smiles>

Ammoniummonofluorselenat.

\section{Fluortellurate.}

Da das Tellur in vieler Beziehung den Metallen, besonders dem Antimon gleicht, schien es möglich, dafs in den Telluraten ebenso leicht wie in den Salzen der Metallsäuren (Antimonsäure, Molybdänsäure und anderer) Sauerstoff durch Fluor ersetzbar sein könnte. Solche fluorierte Tellurate scheinen aber, wenn sie überhaupt existieren, sehr wenig Neigung zum Krystallisieren zu besitzen, wenigstens wurden bei der Einwirkung von Fluorwasserstoffsäure verschiedener Stärke auf Kaliumtellurate, sauere wie basische, immer nur syrupartige Rückstände erhalten, aus denen sich auch bei langem Stehen im Exsiccator kein fester Körper abschied. Nur auf die unten beschriebene Weise, welche nach vielem Experimentieren herausgefunden wurde, gelang es, ein Kialium - und Rubidiumtellurat darzustellen, in welchem 1 Atom Sauerstoff, bezw. 2 Hydroxylgruppen durch 2 Atome Fluor ersetzt sind. Die Frage, ob es nicht fluorreichere Tellurate giebt, bleibt eine offene.

\section{Kaliumdifluortellurat, $\mathrm{TeO}_{3} \mathrm{Fl}_{2} \mathrm{~K}_{2} \cdot 3 \mathrm{H}_{2} \mathrm{O}$.}

Zur Darstellung dieses Salzes fügt man zu einer wässerigen Lösung von 1 Mol. Tellursäure, ${ }^{1} 2$ Mol. Kaliumhydroxyd und dampft auf dem Wasserbad zur Trockne. Den Rückstand Iöst man in .Fluorwasserstoffsäure von etwa $40 \%$ auf und konzentriert dann bei sehr mäfsiger Wärme fast bis zur Syrupdicke. Darauf wird soviel festes Kaliumhydroxyd hinzugefügt, dal's die Lösung nicht mehr viel überschüssige Fluorwasserstoffsäure enthält. Aus dieser durch die Neutralisationswärme heifs gewordenen Flüssigkeit scheidet sich beim Erkalten das Kaliumfluortellurat in Form eines schweren weifsen

${ }^{1}$ Die Tellursäure wurde aus $60 \%$ igem siebenbürgischen Rohtellur nach dem Verfahren von Staudenmaier ( $/$. anorg. Chem. 10, 192. 1895) dargestellt. 
Pulvers aus. Fügt man nun soviel Wasser hinzu, dafs sich das Salz beim Erwärmen gerade wieder löst, und bringt dann die Lösung in einen Exsiccator über Ätzkalk, so erbält man nach kurzer Zeit den Körper in sehr kleinen Krystallen. Es ist bei dieser Darstellungsweise besonders wichtig, dals vor dem Zusatz des Kaliumhydroxyds nicht zuviel überschüssige Fluorwasserstoffsäure vorhanden ist; in diesem Falle erhält man, wohl infolge davon, dafs man mehr Kaliumhydroxyd zusetzen mufs, nur eine Ausscheidung von sauerem Kaliumfluorid und kein Fluortellurat. Fügt man zu wenig Kaliumhydroxyd hinzu, so findet die Ausscheidung des Salzes ebenfalls nicht statt.

Das Kaliumfluortellurat bildet sehr kleine Krystalle. Diese zeigen unter dem Mikroskop oktaëdrischen Habitus und gehören nach freundlicher Mitteilung des Herrn Dr. ZirngiebL wahrscheinlich dem monoklinen System an.

In trockener Luft ist das Salz ziemlich beständig. Erhitzt man es im Reagensglase, so verflüchtigen sich zunächst Wasser und wenig Fluorwasserstoff; bei stärkerem Erhitzen entweicht Sauerstoff und im Rückstand bleibt ein Gemenge (oder Verbindung?) von Tellurdioxyd mit Kaliumfluorid. Hat man nicht lange genug erhitzt, so ist ihm auch noch Tellurat beigemengt. Dieser Rückstand ist, solange er heifs ist, dunkel gefärbt, erkaltet ist er farblos.

Das Kaliumfluortellurat löst sich ziemlich schwer in Wasser zu einer schwach saueren Flüssigkeit. Konzentriert man diese, so erhält man das Salz nicht wieder. Ebensowenig ist es aus Fluorwasserstoffsäure umkrystallisierbar.

\section{Analyse.}

Zur Bestimmung des Tellurs wurden die Fluortellurate mit Salpetersäure bis zur Verflüchtigung sämtlichen Fluorwasserstoffes erwärmt, die Salpetersäure mit Salzsåure beseitigt und dann die Lösung so lange mit der letzteren Säure auf dem Wasserbade erhitzt, bis alle Tellursäure zu telluriger Säure reduziert war; aus dieser Lösung wurde das Tellur mit Ammoniumsulfit gefällt und als solches gewogen. Durch Abdampfen des Filtrats wurde das Alkali als Sulfat erhalten.

Das Fluor wurde wie bei den Phosphaten (S. 45) durch Verflüchtigung als Siliciumfluorid bestimmt. Der Wassergehalt wurde durch Glühen der Salze mit Bleioxyd ermittelt (s. die Einzelheiten bei den Phosphaten S. 46).

I. $0.5964 \mathrm{~g}$ Substanz lieferten $0.2180 \mathrm{~g}$ Te $=36.6 \%$ Te.

Dieselbe Substanz lieferte $0.3050, \mathrm{~K}_{2} \mathrm{SO}_{4}=23.0 \% \mathrm{~K}$.

$0.3148 \mathrm{~g}$ Substanz lieferten $0.0492, \mathrm{H}_{2} \mathrm{O} \quad 15.6 \% \quad \mathrm{H}_{2} \mathrm{O}$.

$0.5244 " \quad " \quad$ nach Abzug von $1.5 \mathrm{mg}$

$0.0825 \mathrm{~g} \mathrm{SiFl}_{4}=11.5 \% \mathrm{Fl}$. 


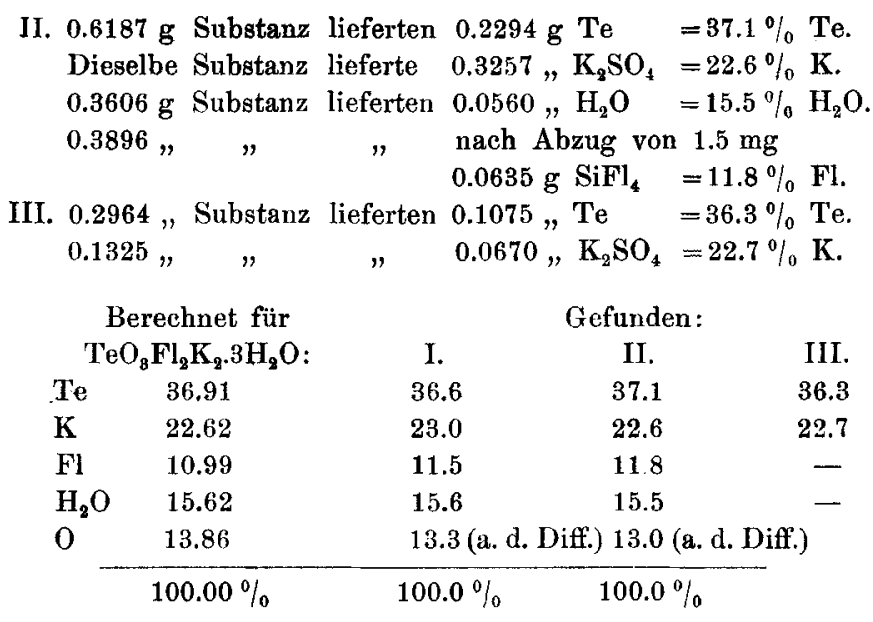

2. Rubidiumdifluortellurat, $\mathrm{TeO}_{3} \mathrm{Fl}_{2} \mathrm{Rb}_{2} \cdot 3 \mathrm{H}_{2} \mathrm{O}$.

Auf dieselbe Weise wie das Kaliumfluortellurat lärst sich auch das diesem entsprechende Rubidiumdifluortellurat darstellen.

Das Rubidiumfluortellurat bildet sehr kleine, farblose Krystalle vom Aussehen des Kaliumsalzes. Es ist in Wasser etwas leichter löslich als jenes; da nun die Rubidiumsalze des Handels meist noch einige Prozente Kaliumsalz enthalten, scheidet sich bei der Darstellung des Rubidiumfluortellurats das vom verunreinigenden Kalium herrührende Kaliumfluortellurat zuerst aus, und die erste Krystallisation giebt bei der Analyse für Tellur und Wasser etwas zu hohe, für das Alkali zu niedrige Werte.

\section{Analyse.}

Salz I war eine erste, Salz II eine spätere, Salz III wiederum eine erste Krystallisation.

I. $0.7152 \mathrm{~g}$ Substanz lieferten $0.2149 \mathrm{~g} \mathrm{Te}=30.1 \% \mathrm{Te}$.

Dieselbe Substanz lieferte $0.4212, \mathrm{Rb}_{2} \mathrm{SO}_{4}=37.7 \% \mathrm{Rb}$.

$0.1276 \mathrm{~g}$ Substanz lieferten $0.0172, \mathrm{H}_{2} \mathrm{O}=13.5 \% \mathrm{H}_{2} \mathrm{O}$.

$0.2314, \quad " \quad, \quad$ nach Abzug von $1.5 \mathrm{mg}$

$0.0246 \mathrm{~g} \mathrm{SiFl}_{4}=7.7 \% \mathrm{Fl}$.

II. $0.3259 \mathrm{~g}$ Substanz lieferten $0.0955, \mathrm{Te}=29.3 \% \mathrm{Te}$

Dieselbe Substanz lieferte $0.1974, \mathrm{Rb}_{8} \mathrm{SO}_{4}=38.8 \% \mathrm{~Kb}$.

$0.3086 \mathrm{~g}$ Substanz lieferten $0.0400, \mathrm{H}_{2} \mathrm{O}=13.0 \% \mathrm{H}_{2} \mathrm{O}$.

$0.5484, \quad " \quad, \quad$ nach Abzug von $1.5 \mathrm{mg}$

$0.0616 \mathrm{~g} \mathrm{SiFl}_{4}=8.2 \% \mathrm{Fl}$.

III 0.2372 "Substanz lieferten 0.0719, Te $\quad=30.3 \%$ Te. 


\begin{tabular}{lcccc}
\multicolumn{2}{c}{ Berechnet für } & \multicolumn{3}{c}{ Gefunden: } \\
$\mathrm{TeO} \mathrm{Fl}_{2} \mathrm{Rb}_{2} .3 \mathrm{H}_{2} \mathrm{O}:$ & I. & II. & III. \\
$\mathrm{Te}$ & 29.12 & 30.1 & 29.3 & 30.3 \\
$\mathrm{Rb}$ & 38.96 & 37.7 & 38.8 & - \\
$\mathrm{Fl}$ & 8.66 & 7.7 & 8.2 & - \\
$\mathrm{H}_{2} \mathrm{O}$ & 12.32 & 13.5 & 13.0 & - \\
$\mathrm{O}$ & 10.94 & 10.0 (a. d. Diff.) 10.7 (a. d. Diff.)
\end{tabular}

Ein Fluortellurat vom Ammonium oder Natrium liels sich auf die angegebene Weise nicht darstellen.

Wie man das Ammoniumfluorselenat als durch Anlagerung von 1 Mol. Fluorwasserstoff an 1 Mol. Ammoniumselenat entstanden sich vorstellen kann, so lassen sich das Kalium- und Rubidiumdifluortellurat als durch Anlagerung von $2 \mathrm{Mol}$. Fluorwasserstoff an 1 Mol. Kalium-bezw. Rubidiumtellurat unter Aufrichtung von beiden an das Tellur doppelt gebundenen Sauerstoffatomen gebildet ansehen; die Salze enthalten dann 2 Mol. Krystallwasser:<smiles>[H][R]([H])(O)(O)(O)O</smiles>

Ammoniummonofluorselenat,

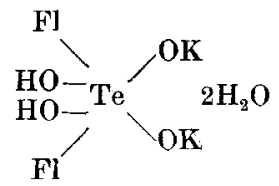

Kaliumdifluortellurat.

Das Tellur wäre demnach zur Aufnahme von Fluor geneigter als Selen und Schwefel, was seiner mehr metallischen Natur zuzuschreiben wäre.

Man kann aber auch die Difluortellurate als Tellurate auffassen, in denen 1 Atom Sauerstoff durch 2 Atome Fluor vertreten ist:

$$
\mathrm{TeO}_{3} \mathrm{Fl}_{2} \mathrm{Me}_{2} \cdot 3 \mathrm{H}_{2} \mathrm{O} \text {. }
$$

Welches Sauerstoffatom dies ist, bleibt fraglich. Von den

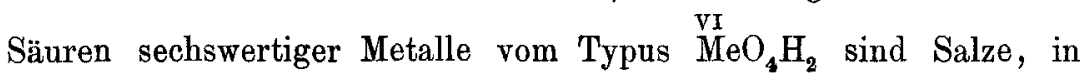
welchen nur ein Sauerstoffatom durch Fluor ersetzt ist, bis jetzt nicht bekannt: Von den Fluormolybdänaten und Fluorwolframaten kommen die Formen $\mathrm{Mo}(\mathrm{W}) \mathrm{O}_{2} \mathrm{Fl}_{4} \stackrel{\mathrm{I}}{\mathrm{M}} \mathrm{e}_{2} \cdot \mathrm{H}_{2} \mathrm{O} ; \mathrm{Mo}(\mathrm{W}) \mathrm{O}_{2} \mathrm{Fl}_{3} \stackrel{\mathrm{I}}{\mathrm{Me}}$. $\mathrm{H}_{2} \mathrm{O}$ und $\mathrm{MoOFl}_{5} \mathrm{Me}$ vor. Den Fluorchromaten liegt die Fluorchromsäure $\mathrm{FlCrO}_{3} \mathrm{H}$ zu Grunde. Die Fluoruranate zeigen die durch die Formel $\mathrm{UO}_{2} \mathrm{Fl}_{5} \mathrm{Me}_{3}$ ausgedrückte Zusammensetzung. 


\section{E. Fluordithionate.}

Durch Einwirkung von Fluorwasserstoffsäure liefs sich in das Kalium-, Rubidium- und Cäsiumdithionat Fluor einführen, und zwar traten bei den beiden ersteren 2 Atome Fluor für zwei Hydroxylgruppen, beim letzteren 1 Atom Fluor für eine Hydroxylgruppe ein.

1. Kaliumdifluordithionat, $\mathrm{S}_{2} \mathrm{O}_{5} \mathrm{Fl}_{2} \mathrm{~K}_{2} \cdot 3 \mathrm{H}_{2} \mathrm{O}$.

Dieses Salz scheidet sich aus einer in der Wärme gesättigten Lösung von Kaliumdithionat in etwa $40 \%$ iger Fluorwasserstoffsäure beim Erkalten in schönen Krystallen aus. Auch bei Anwendung von $60 \%$ iger Fluorwasserstoffsäure erhält man es. Diese darf indessen nicht bis zum Sieden erhitzt werden, da sich sonst das Dithionat unter Entwickelung von Schwefeldioxyd zersetzt. War die Lösung nicht genügend gesättigt, so krystallisiert bei längerem Stchen derselben im Exsiccator fluorfreies Kaliumdithionat aus.

Uber die Krystallform des Salzes teilt uns Herr Dr. ZIRngIebL folgendes freundlichst mit:

„Die Krystalle sind nicht melsbar. Sie sind tafelig, spielsig und Zwillinge, welche auf der tafeligen Fläche eine Axe schief austretend zeigen. Einspringender Winkel an der schmalen Seite der Länge nach verlaufend. Keine deutlichen Endflächen. Die Krystalle gleichen im Aussehen den tafeligen Titanitzwillingen, sind aber vielleicht triklin."

Das Kaliumfluordithionat ist sehr unbeständig. Lälst man die mit Filtrierpapier von der Mutterlauge befreiten Krystalle an der Luft liegen, so werden sie rasch tribe und zerfallen in kurzer Zeit unter Verlust sämtlichen Wassers und Fluors (als Fluorwasserstoff) zu einem weifsen Pulver (Kaliumdithionat). Auch in Paraffin bleiben sie nur kurze Zeit unverändert, und es tritt namentlich an den Stellen, wo sie mit dem Glas in Berührung kommen, rasch Zersetzung ein. Erhitzt man das Salz im Reagensrohr, so verflüchtigen sich zuerst Wasser und Fluorwasserstoff; bei stärkerem Erhitzen entweicht dann Schwefeldioxyd, und Kaliumsulfat bleibt im Rückstand. In Wasser löst sich das Salz leicht unter Abspaltung sämtlichen Fluors als Fluorwasserstoff.

$$
\text { Analyse. }
$$

Da die Fluordithionate sich sehr rasch zersetzen, müssen sie sogleich analysiert werden, nachden sie mittels Filtrierpapiers von der Mutterlauge befreit sind und ganz kurze Zeit im Exsiccator gelegen haben. 
Das Alkali wurde durch Abrauchen der Salze mit wenig konz. Schwefelsäure als Sulfat bestimmt.

Um den Schwefelgehalt festzustellen, wurde die wässerige Lösung der Salze mehrmals mit rauchender Salpetersäure auf dem Wasserbade eingedampft und die gebildete Schwefelsäure mit Baryumehlorid gefällt.

Das Flu or wurde durch Titration der bei der Zersetzung der Salze durch Wasser gebildeten freien Säure bestimmt; da den Salzen neutrales Dithionat zu Grunde liegt, kommt der ganze Säuregehalt der wässerigen Lösung auf Rechnung des Fluorwasserstoffes. - Aufserdem wurde das Fluor nach der Methode von $H$. Rose ${ }^{1}$ als Calciumfluorid gefällt und gewogen.

Der Wassergehalt wurde wie bei den anderen Salzen durch Erhitzen mit Bleioxyd ermittelt.

I. $0.4208 \mathrm{~g}$ Substanz lieferten $0.2340 \mathrm{~g} \mathrm{SO}_{4} \mathrm{~K}_{2}=24.9 \% \mathrm{~K}$. $0.5287, \quad " \quad \cdots \quad 0.7927, \mathrm{SO}_{4} \mathrm{Ba}=20.6 \% \mathrm{~S}$. $0.6086, " \quad$ verbrauchten zur: Bindung der freien Säure der wässerigen Lösung $40 \mathrm{~cm} 1 / 10$-norm. Kalilauge $=12.5 \% \mathrm{Fl}$. $0.8490 \mathrm{~g}$ Substanz lieferten $0.2068 \mathrm{~g} \mathrm{CaFl}_{2}=11.9 \% \mathrm{Fl}$. $0.3808, " \quad " \quad 0.0646, \mathrm{H}_{2} \mathrm{O}=17.0 \% \mathrm{H}_{2} \mathrm{O}$.

II. 0.4600 "Substanz lieferten $0.2539, \mathrm{~K}_{2} \mathrm{SO}_{4}=24.8 \% \mathrm{~K}$. $0.6006 " \quad " \quad " \quad 0.8807, \mathrm{BaSO}_{4}=20.1 \% \mathrm{~S}$. $1.0836 " \quad " \quad, \quad 0.2689 " \mathrm{CaFL}_{2}=12.1 \% \mathrm{Fl}$. $1.0193 " \quad " \quad 0.1720, \mathrm{H}_{2} \mathrm{O}=16.9 \% \mathrm{H}_{2} \mathrm{O}$.

III. 0.3314 , Substanz verbrauchten zur Bindung der freien Säure der wässerigen Lösung $21.0 \mathrm{ccm} 1 / 10^{-n o r m}$. Kalilauge

$$
=12.03 \% \mathrm{Fl} \text {. }
$$

IV. $0.4426 \mathrm{~g}$ Substanz verbrauchten ebenso $28.5 \mathrm{ccm}{ }^{1 / 1} 0^{*}$ norm. Kalilauge $=12.22 \% \mathrm{Fl}$.

V. 0.3462 "Substanz verbrauchten ebenso $22.4 \mathrm{cem} 1 / 10^{- \text {norm. Kalilauge }}$ $=12.31 \%$ FI.

Berechnet für

\begin{tabular}{lcccccr} 
& $\mathrm{S}_{2} \mathrm{O}_{5} \mathrm{Fl}_{2} \mathrm{~K}_{2} .3 \mathrm{H}_{2} \mathrm{O}:$ & I. & II. & III. & IV. & V. \\
$\mathrm{S}$ & 20.39 & 20.6 & 20.1 & - & - & - \\
$\mathrm{K}$ & 24.88 & 24.9 & 24.8 & - & - & - \\
$\mathrm{Fl}$ & 12.11 & 11.9 & 12.1 & 12.03 & 12.22 & 12.31 \\
$\mathrm{H}_{2} \mathrm{O}$ & 17.18 & 17.0 & - & - & - & - \\
$\mathrm{O}$ & 25.44 & \multicolumn{2}{c}{25.6 (a. d. Diff.) } & & &
\end{tabular}

2. Rubidiumdifluordithionat, $\mathrm{S}_{2} \mathrm{O}_{5} \mathrm{Fl}_{2} \mathrm{Rb}_{2} \cdot 3 \mathrm{H}_{2} \mathrm{O}$.

Das Rubidiumdifluordithionat entspricht nach Bildung, Form und Eigenschaften völlig dem beschriebenen Kaliumdifluordithionat. (Das zu den Versuchen benutzte Rubidiumdithionat wurde durch Einwirkung von Rubidiumsulfat auf Baryumdithionat dargestellt.)

${ }^{1}$ S. Fresenius, Quantit. Analyse (6. Aufl.) 1, 429.

Z. anorg. Chem. XXI. 
Analyse.

$0.5057 \mathrm{~g}$ Substanz lieferten $0.3332 \mathrm{~g} \mathrm{Rb_{2 }} \mathrm{SO}_{4}=42.3 \% \mathrm{Rb}$.

$0.3094 " \quad " \quad 0.3696, \mathrm{BaSO}_{4}=16.2 \% \mathrm{~S}$.

$0.2030 ", " \quad " \quad 0.0266 \cdot, \mathrm{H}_{2} \mathrm{O}=13.1 \% \mathrm{H}_{2} \mathrm{O}$.

$0.5677, \quad$ verbrauchten zur Bindung der Säure der wässerigen Lösung $28.2 \mathrm{ccm} 1 / 10^{-n o r m}$. Kalilauge $=9.4 \% \mathrm{Fl}$.

Berechnet für $\mathrm{S}_{2} \mathrm{O}_{5} \mathrm{Fl}_{2} \mathrm{Rb}_{2}, 3 \mathrm{H}_{2} \mathrm{O}$ :

\begin{tabular}{lrc}
$\mathrm{S}$ & 15.75 & 16.2 \\
$\mathrm{Rb}$ & 41.97 & 42.3 \\
$\mathrm{Fl}$ & 9.36 & 9.4 \\
$\mathrm{H}_{2} \mathrm{O}$ & 13.27 & 13.1 \\
$\mathrm{O}$ & 19.65 & 19.0 (a. d. Diff.) \\
\cline { 2 - 3 } & $100.00 \%$ & $100.0 \%$
\end{tabular}

3. Cäsiummonofluorditbionat, $\mathrm{S}_{2} \mathrm{O}_{5}(\mathrm{OH}) \mathrm{FlCs}_{2} \cdot \mathrm{H}_{2} \mathrm{O}$.

Sättigt man, wie bei der Darstellung des Kalium- und Rubidiumdifluordithionats, heifse Fluorwasserstoffsäure mit Cäsiumdithionat, ${ }^{1}$ so scheidet sich beim Erkalten Cäsiummonofluordithionat aus. Wie dieses Salz nur die Hälfte des Fluors des Kalium- und Rubidiumdifluordithionats enthält, so ist auch sein Wassergehalt nur halb so grols als der jener Salze.

Das Cäsiummonofluordithionat bildet farblose, kleine, dicke Prismen. Es ist etwas weniger unbeständig als das Kalium- und Rubidiumsalz, verbält sich aber im übrigen wie jene.

\section{Analyse.}

I. $0.5616 \mathrm{~g}$ Substanz verbrauchten zur Bindung der Säure der wässerigen Lösung $12 \mathrm{ccm} 1 / 10$-norm. Kalilange $=4.06 \% \mathrm{Fl}$. Dieselbe Substanz lieferte $0.5524 \mathrm{~g} \mathrm{BaSO}_{4}=13.5 \% \mathrm{~S}$. $0.4450 \mathrm{~g}$ Substanz lieferten $0.0298, \mathrm{H}_{2} \mathrm{O}=6.7 \% \mathrm{H}_{2} \mathrm{O}$.

II. 0.3800 "Substanz verbrauchten zur Bindung der Säure der wässerigen Lösung $8.4 \mathrm{ccm} 1 / 10$-norm. Kalilauge $=4.19 \% \mathrm{Fl}$. Dieselbe Substanz lieferte $0.3880 \mathrm{~g} \mathrm{BaSO}_{4}=14.0 \% \mathrm{~S}$. $0.2335 \mathrm{~g}$ Substanz lieferten $0.1821, \mathrm{Cs}_{2} \mathrm{SO}_{4}=57.3 \%$ Cs. $0.5046 " \quad " \quad 0.3050, \mathrm{H}_{2} \mathrm{O}=6.0 \% \mathrm{H}_{2} \mathrm{O}$.

Berechnet für

\begin{tabular}{lccc}
\multicolumn{2}{c}{$\mathrm{S}_{2} \mathrm{O}_{5}(\mathrm{OH}) \mathrm{FlCs}_{2} \cdot \mathrm{H}_{2} \mathrm{O}:$} & $\mathrm{I}$. & III. \\
$\mathrm{S}$ & 13.83 & 13.5 & 14.0 \\
$\mathrm{Cs}$ & 57.29 & - & 57.3 \\
$\mathrm{Fl}$ & 4.09 & 4.06 & 4.19 \\
$\mathrm{H}_{3} \mathrm{O}$ & 5.82 & 6.7 & 6.0 \\
$\mathrm{O}$ & 18.97 & - & 18.51 (a. d. Diff.) \\
\cline { 2 - 4 } & $100.00 \%$ & & $100.0 \%$
\end{tabular}

1 Dieses wurde durch Einwirkung von Cäsiumsulfat auf Baryumdithionat dargestellt. 
Natrium- und Ammoniumdithionat liefsen sich auf die beschriebene Weise nicht fluorieren.

Die Versuche, auch in die Salze der höheren Polythionsäuren Fluor einzuführen, scheiterten daran, dafs die Fluorwasserstoffsäure auf diese Salze zersetzend einwirkt (nachgewiesen beim Kaliumtrithionat und Kaliumtetrathionat).

Bei den Fluordithionaten gelangt man, wie bei den Fluorphosphaten, -sulfaten, -selenaten und -telluraten, durch die Annahme einer Anlagerung von Fluorwasserstoff unter Aufrichtung von ein oder zwei doppelt an den Schwefel gebundenen Sauerstoffatomen zu einer einfachen Vorstellung ihres Aufbaues. Bei dem fluorierten Cäsiumsalz der Dithionsäure, welche jetzt ziemlich allgemein als aus zwei direkt gebundenen Sulfogruppen bestehend angenommen wird, hat sich demgemäls an eine Sulfogruppe 1 Mol. Fluorwasserstoff, bei dem Kalium- und Rubidiumsalz an jede Sulfogruppe je 1 Mol. Fluorwasserstoff unter Hydroxylierung von 1 bezw. 2 Sauerstoffatomen angelagert. Aufserdem haben sich beim Kalium- und Rubidiumsalz beide noch vorhandenen, doppelt an den Schwefel gebundenen Sauertoffatome, beim Cäsiumsalz jedoch nur ein solches Sauerstoffatom durch Hinzutritt von Wasser hydroxyliert.

Anlagerung von Fluorwasserstoff und von Wasser scheinen demnach in diesem Fall einander parallele Vorgänge zu sein.

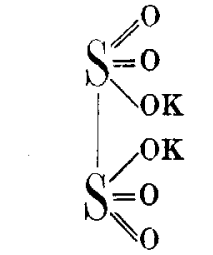

Kaliundithionat,

$\mathrm{S}_{2} \mathrm{O}_{6} \mathrm{~K}_{2}$.

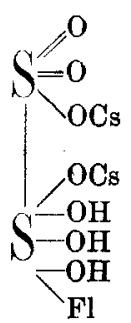

Cäsiummonofluordithionat, $\mathrm{S}_{2} \mathrm{O}_{5}(\mathrm{OH}) \mathrm{FlCs}_{2} \cdot \mathrm{H}_{2} \mathrm{O} .^{1}$

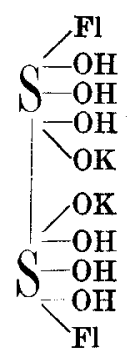

Kaliumdifluordithionat, $\mathrm{S}_{2} \mathrm{O}_{5} \mathrm{Fl}_{2} \mathrm{~K}_{2} \cdot 3 \mathrm{H}_{2} \mathrm{O} .^{1}$

\section{Zusammenfassung.}

Bei den beschriebenen Fluorphosphaten, Fluorsulfaten, Fluorselenaten, Fluortelluraten und Fluordithionaten gelangt man, wie schon ausgeführt, durch die Annahme, dals sie durch

${ }^{1} \mathrm{Im}$ Texte wurden die Formeln $\mathrm{S}_{2} \mathrm{O}_{5} \mathrm{Fl}_{2} \mathrm{~K}_{2}\left(\mathrm{Rb}_{4}\right) \cdot 3 \mathrm{H}_{2} \mathrm{O}$ benutzt, damit die Dithionatform leichter kenntlich bleibt. 
Anlagerung von Fluorwasserstoff an die betreffenden, teils saueren, teils neutralen Salze unter Aufrichtung doppelt gebundener Sauerstoffatome entstanden sind, zu einer einfachen und einheitlichen Vorstellung über ihre Konstitution. Sie erscheinen so als mehr oder weniger vollständig hydroxylierte Salze, in denen eine oder mehrere Hydroxylgruppen durch Fluor ersetzt sind.

Es haben sich somit, immer unter Hydroxylierung doppelt gebundener Sauerstoffatome, angelagert:

1. Bei den primären Monofluorphosphaten (S. 43) 1 Mol. Fluorwasserstoff an 1 Mol, primäres Phosphat:<smiles>[O-][P+]([O-])(O)O</smiles>

Primäres Phosphat.<smiles>COP(O)(O)(O)O</smiles>

Monofluorphosphat.

2. Bei den saueren Fluorsulfaten (S. 50) 2 Mol. Fluorwasserstoff an $1 \mathrm{Mol}$. saueres Sulfat der Formel $\left(\mathrm{SO}_{4}\right)_{2} \mathrm{HMe}_{3}$ :<smiles>COS1(=O)(O)OS(=O)(O)(OC)O1</smiles>

Saueres Sulfat $\left(\mathrm{SO}_{4}\right)_{2} \mathrm{HMe}_{3}$.

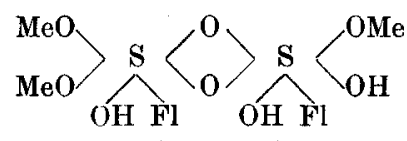

Difluordisulfat.

3. Bei dem saueren Kalium- und Rubidiumfluorselenat (S. 55), wie bei den Fluorsulfaten 2 Mol. Fluorwasserstoff an 1 Mol. des saueren Selenats der Formel $\left(\mathrm{SeO}_{4}\right)_{2} \mathrm{HK}_{3}\left(\mathrm{Rb}_{3}\right)$ :<smiles>CCCCO[Se]1(=O)O[Se](=O)(O[14CH3])O1</smiles>

Saueres Selenat $\left(\mathrm{SeO}_{4}\right)_{2} \mathrm{HK}_{3}\left(\mathrm{Rb}_{8}\right)$.

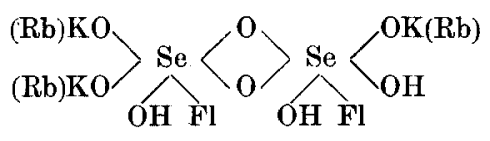

Difluordiselenat.

4. Beim Ammoniummonofluorselenat (S. 58) 1 Mol. Fluorwasserstoff an 1 Mol. neutrales Ammoniumselenat:<smiles>NO[Se](=O)(O)ON</smiles>

Ammoniumselenat.<smiles>NO[SbH](O)O</smiles>

Ammoniummonofluorselenat. 
5. Bei den Difluortelluraten (S. 60) 2 Mol. Fluorwasserstoff an 1 Mol. normales Tellurat:<smiles>CO[14C](=O)OC</smiles>

Normales Tellurat.<smiles>[2H][C@@H](O)C(OC)(OC)OC</smiles>

Difluortellurat.

6. Beim Cäsiummonofluordithionat (S. 66) 1 Mol. Fluorwasserstoff und $1 \mathrm{Mol}$. Wasser an $1 \mathrm{Mol}$. Cäsiumdithionat:<smiles>O=S(=O)(O[Na])O[Na]</smiles>

Cäsiumdithionat.<smiles>CCCOC(O)(O)C(O)(O)C(=O)O</smiles>

Cäsiummonofluordithionat.

7. Beim Kalium- und Rubidiumdifluordithionat (S. 64) 2 Mol. Fluorwassertoff und 2 Mol. Wasser an 1 Mol. Dithionat:<smiles>COS(=O)(=O)S(=O)(=O)OC</smiles>

Dithionat.<smiles>CO[C@H](O)[C@@H](O)[C@@H](O)C(O)O</smiles>

Difluordithionat.

Die schon früher beobachtete Ähnlichkeit des Fluors mit dem Sauerstoff wird durch die vorliegende Untersuchung insofern aufs neue bestätigt, als auch der Fluorwasserstoff, gleichwie das Wasser, zur Aulagerung an verschiedene Salze befähigt ist, an welche eine Anlagerung der. anderen Halogenwasserstoffe bis jetzt nicht nachgewiesen wurde. Der Fluorwasserstoff zeigt somit, trotz seines saueren Charakters, in dieser Beziehung eine gewisse Ähnlichkeit mit dem Wasser.

Mïnchen, Labor. für angew. Chemie der kgl. Universität, 22. Mär\% 1899.

Bei der Redaktion eingegangen am 23. März 1899. 